\title{
When the Diagnosis is a Victim of the Circumstances
}

\author{
$\underline{\text { João Afonso Rodrigues }}{ }^{1}$, Pedro Brogueira ${ }^{2}$, Sara Rodrigues ${ }^{3}$, Margarida Cardoso ${ }^{2}$, Tiago Pack ${ }^{1}$ \\ ${ }^{1}$ Internal Medicine, Centro Hospitalar de Lisboa Central, Lisbon, Portugal \\ ${ }^{2}$ Infectious Disease, Centro Hospitalar de Lisboa Ocidental, Lisbon, Portugal \\ ${ }^{3}$ Internal Medicine, Centro Hospitalar de Lisboa Norte, Lisbon, Portugal
}

Received: 07/02/2017

Accepted: $16 / 02 / 2018$

Published: 20/03/2018

How to cite this article: Rodrigues JA, Brogueira P, Rodrigues S, Cardoso M, Pack T. When the diagnosis is a victim of the circumstances. EJCRIM 2018;5: doi:10.12890/2018_000862.

Conflicts of Interests: The Authors declare that there are no competing interests.

This article is licensed under a Commons Attribution Non-Commercial 4.0 License

\section{ABSTRACT}

Introduction: Haemoptysis is a common symptom which can sometimes mimic gastrointestinal bleeding.

Case description: We describe the case of a 31-year-old man who presented to the emergency department after an episode of sudden nausea and presumed massive haematemesis. The situation was interpreted as gastrointestinal bleeding but clinical evolution and greater attention to the anamnesis resulted in a diagnosis of pulmonary tuberculosis and the provision of appropriate care.

Discussion: This report emphasizes the difficulty of differentiating between haemoptysis and haematemesis and the importance of a careful anamnesis and attention to all clinical circumstances for an accurate diagnosis.

\section{LEARNING POINTS}

- Haematemesis and haemoptysis are not always distinguished from each other when a patient's history is being collected.

- Despite being a rare manifestation of tuberculosis, in the correct epidemiological context, haemoptysis should raise the suspicion of pulmonary tuberculosis.

- The clinical setting and the need for immediate care should not limit clinical investigation or the differential diagnosis.

\section{KEYWORDS}

Tuberculosis, haemoptysis, haematemesis

\section{INTRODUCTION}

Haemoptysis is a common symptom and can range from blood-streaking of sputum to massive haemoptysis associated with significant mortality (approximately $80 \%$ ) ${ }^{[1]}$. Sometimes haemoptysis can mimic upper gastrointestinal bleeding, thus leading to serious misdiagnosis. Although most cases are benign, self-limiting events, the presentation of haemoptysis may be a sign of major underlying tracheopulmonary pathology. 


\section{CASE REPORT}

The authors present the case of a 31-year-old athletic African man on holidays in Europe. He presented to the emergency department with an episode of sudden nausea and massive haematemesis while drinking beer in a bar. He mentioned occasional mild epigastric discomfort over the previous few months, intensified with food intake. On evaluation, he was hypotensive and tachycardic with abdominal tenderness. A moderate amount $(500 \mathrm{ml})$ of blood was drained from a nasogastric tube after tube placement. Laboratory tests revealed anaemia and mild elevation of the inflammatory parameters, while an arterial blood gas test revealed elevated lactate levels (Table 1).

\begin{tabular}{|c|c|c|c|}
\hline Variable & $\begin{array}{l}\text { Reference range } \\
\text { in adults }\end{array}$ & On presentation & $\begin{array}{l}24 \text { Hours } \\
\text { after admission }\end{array}$ \\
\hline Haemoglobin (g/dl) & $13.0-17.0$ & 11.8 & 11.2 \\
\hline Haematocrit (\%) & $0.406-0.504$ & 0.374 & 0.342 \\
\hline White cell count $(\times 109 / 1)$ & $4.0-10.0$ & 14,900 & 7.8 \\
\hline \multicolumn{4}{|l|}{ Differential count (\%) } \\
\hline -Neutrophils & $40.0-80.0$ & 50.1 & 79.9 \\
\hline -Lymphocytes & $20.0-40.0$ & 37.3 & 9.9 \\
\hline - Monocytes & $2.0-11.7$ & 10.0 & 8.1 \\
\hline - Eosinophils & $1.0-6.0$ & 2.0 & 1.8 \\
\hline -Basophils & $0.0-0.2$ & 0.6 & 0.3 \\
\hline Platelet count $\left(\times 10^{9} / \mathrm{l}\right)$ & $150-400$ & 406 & 212 \\
\hline Sodium (mmol/l) & $136-145$ & 138 & 138 \\
\hline Potassium (mmol/l) & $3.50-5.10$ & 4.17 & 4.26 \\
\hline Creatinine (mg/dl) & $0.70-1.20$ & 1.02 & 0.87 \\
\hline Urea (mg/dl) & $13-43$ & 31 & 22 \\
\hline $\begin{array}{l}\text { Alanine aminotransferase } \\
\text { (U/I) }\end{array}$ & $<41$ & 18 & 17 \\
\hline $\begin{array}{l}\text { Aspartate } \\
\text { aminotransferase (U/I) }\end{array}$ & $<40$ & 26 & 21 \\
\hline $\begin{array}{l}y \text {-Glutamyl transferase } \\
\text { (U/I) }\end{array}$ & $10-71$ & 36 & 30 \\
\hline $\begin{array}{l}\text { Lactate dehydrogenase } \\
\text { (U/I) }\end{array}$ & $135-225$ & 197 & - \\
\hline C-reactive protein $(\mathrm{mg} / \mathrm{dl})$ & $<0.50$ & 3.73 & - \\
\hline
\end{tabular}

The patient was assumed to have hypovolemic haemorrhagic shock, secondary to upper gastrointestinal bleeding, so intense fluid resuscitation was started as well as blood and proton pump inhibitor perfusion. Urgent endoscopy was performed, which detected traces of blood in the stomach but no potentially haemorrhagic lesions. After haemodynamic stabilization, the patient was admitted to a medical ward for surveillance. During the first 24 hours after admission, the patient developed a high fever $\left(39^{\circ} \mathrm{C}\right)$ and dyspnoea at rest with a respiratory rate of $32 \mathrm{bpm}$. On chest examination there were bilateral bronchial sounds and crackles; pulse oximetry showed low oxygen saturation. A chest radiograph (Fig. 1) revealed signs of collapse of the left lung, consolidation in the inferior half and cavitation in the superior lobe of the right lung.

In view of the clinical deterioration and radiological findings, the patient's clinical history was reviewed. He had a previous history of a 30-day stay in hospital 2 years previously for a respiratory infection and in the last 6 months had experienced night sweats and vespertine fever, fatigue for progressively less effort and anorexia with subsequent unintentional weight loss. He had no other relevant medical or surgical history and no significant family medical history. He had no regular medication intake. He did not smoke tobacco or use illicit drugs, consumed alcohol in a social context, but had regular consumption of anabolic drugs (in the last 2-3 years).

A probable diagnosis of pulmonary tuberculosis with respiratory failure was assumed and the patient was immediately transferred to the Intensive Care Unit where he started antituberculosis drugs (isoniazid, rifampicin, pyrazinamide, ethambutol and streptomycin), steroids 
and high frequency oscillatory ventilation. Further examination confirmed the diagnosis. Chest computed tomography (Fig. 2) revealed multiple areas of cavitation in the left lung and a dominant area of cavitation in the upper right lobe of the right lung, while mycobacterial examination of sputum (with Ziehl-Neelsen staining) was positive for acid-alcohol resistant bacilli, and culture examination was positive after 8 days for Mycobacterium tuberculosis complex.

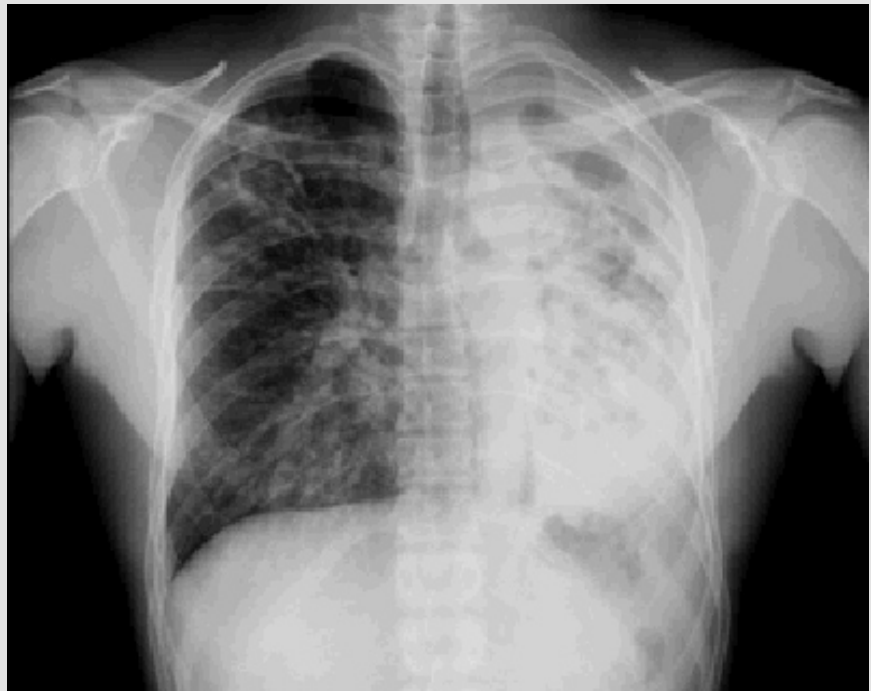

Figure 1. Chest $x$-ray showing collapse of the left lung, consolidation in the inferior half and suspected cavitation in the superior right lobe of the right lung

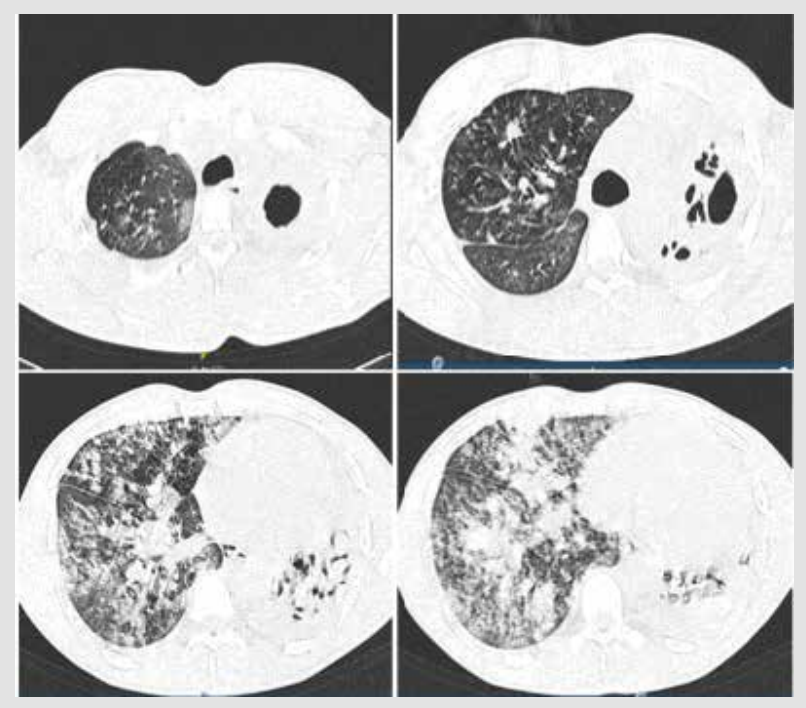

Figure 2. Computerized tomography of the chest showing pulmonary collapse to the left and a shift of the mediastinum to the left, with evidence of multiple areas of cavitation in the left lung, with no intracavitary content. A large cavity about $3.3 \mathrm{~cm}$ in diameter with intracavitary vegetation was seen in the upper right lobe of the right lung, in addition to several nodular opacities

The patient gradually improved and was discharged from hospital after 30 days, maintaining tuberculosis chemotherapy and clinical followup in the outpatient clinic.

\section{DISCUSSION}

Tuberculosis is the ninth leading cause of death worldwide ${ }^{[2]}$, with more than two billion people estimated to be infected with $M$. tuberculosis ${ }^{[3]}$. Failure or delay in obtaining the correct diagnosis contributes to more severe disease at presentation ${ }^{[4]}$. As the symptoms of pulmonary tuberculosis are non-specific, a high index of suspicion is essential to prompt diagnosis and treatment, especially in the relevant epidemiological context.

In the present case, the delayed diagnosis resulted from many misconceptions. Vomiting blood after alcohol ingestion associated with mild epigastric pain strongly suggested upper gastrointestinal bleeding that, in this case, was also supported by the moderate blood drainage from the nasogastric tube. The haemodynamic instability prompted immediate intervention without a careful anamnesis, which consequently delayed a definitive diagnosis.

The meticulous patient history and the epidemiological context suggest this patient had a previous primary pulmonary tuberculosis that was treated as bacterial pneumonia, leading to subclinical infection that evolved with lung cavitation and persistent local inflammation. Reactivation and mild constitutional symptoms were masked by the patient's athletic constitution and the androgen-anabolic steroid consumption. Vascular inflammation eventually resulted in vessel erosion and alveolar haemorrhage. Vomiting of swallowed blood of bronchopulmonary origin can be easily confused with haematemesis which could explain our patient's atypical presentation and the finding of blood in the nasogastric drainage. Haemoptysis has multiple causes with a broad differential: bronchitis, bronchogenic carcinoma and bronchiectasis are the most common causes of haemoptysis in developed countries, while infections due to M. tuberculosis and Paragonimus westermani are more common causes in endemic countries ${ }^{[4]}$. 
Consequently, a diagnosis of pulmonary tuberculosis presenting with moderate haemoptysis should have been considered as highly probable in the initial approach.

In conclusion, the presented case emphasizes the difficulty in differentiating haemoptysis from haematemesis and the importance of a careful anamnesis for accurate diagnosis.

\section{REFERENCES}

1. Dweik RA, Stoller JK. Role of bronchoscopy in massive hemoptysis. Clin Chest Med 1999;20:89-105

2. Dheda K, Barry CE 3rd, Maartens G. Tuberculosis. Lancet 2016;387:1211-1226.

3. Storla DG, Yimer S, Bjune GA. A systematic review of delay in the diagnosis and treatment of tuberculosis. BMC Public Health 2008;8:15.

4. Prasad R, Garg R, Singhal S, Srivastava P. Lessons from patients with hemoptysis attending a chest clinic in India. Ann Thorac Med 2009;4:10-12. 